Research article

\title{
A combined approach exploring gene function based on Worm-Human Orthology Ivica Tamas ${ }^{1}$, Emily Hodges ${ }^{2}$, Patrick Dessi ${ }^{3}$, Robert Johnsen ${ }^{4}$ and Ana Vaz Gomes*2
}

Address: ${ }^{1}$ Department of Molecular Biology and Functional Genomics, Stockholm University, Sweden, ${ }^{2}$ Center for Genomics and Bioinformatics, Karolinska Institute, Stockholm, Sweden, ${ }^{3}$ Södertörn University College, Stockholm, Sweden and ${ }^{4}$ Department of Molecular Biology and Biochemistry, Simon Fraser University, Burnaby, Canada

Email: Ivica Tamas - ivica.tamas@molbio.su.se; Emily Hodges - emily.hodges@cgb.ki.se; Patrick Dessi - patrick.dessi@industry.gov.au; Robert Johnsen - bjohnsen@gene.mbb.sfu.ca; Ana Vaz Gomes* - ana.vaz.gomes@cgb.ki.se

* Corresponding author

Published: 06 May 2005

BMC Genomics 2005, 6:65 doi:10.1 186/147|-2/64-6-65
Received: 22 December 2004

Accepted: 06 May 2005

This article is available from: http://www.biomedcentral.com/I47/-2/64/6/65

(c) 2005 Tamas et al; licensee BioMed Central Ltd.

This is an Open Access article distributed under the terms of the Creative Commons Attribution License (http://creativecommons.org/licenses/by/2.0), which permits unrestricted use, distribution, and reproduction in any medium, provided the original work is properly cited.

\begin{abstract}
Background: Many aspects of the nematode Caenorhabditis elegans biology are conserved between invertebrates and vertebrates establishing this particular organism as an excellent genetic model. Because of its small size, large populations and self-fertilization of the hermaphrodite, functional predictions carried out by genetic modifications as well as RNAi screens, can be rapidly tested.
\end{abstract}

Results: In order to explore the function of a set of $C$. elegans genes of unknown function, as well as their potential functional roles in the human genome, we performed a phylogenetic analysis to select the most probable worm orthologs. A total of $13 \mathrm{C}$. elegans genes were subjected to downregulation via RNAi and characterization of expression profiles using GFP strains. Previously unknown distinct expression patterns were observed for four of the analyzed genes, as well as four visible RNAi phenotypes. In addition, subcellular protein over-expression profiles of the human orthologs for seven out of the thirteen genes using human cells were also analyzed.

Conclusion: By combining a whole-organism approach using $C$. elegans with complementary experimental work done on human cell lines, this analysis extends currently available information on the selected set of genes.

\section{Background}

Many aspects of the nematode Caenorhabditis elegans biology are conserved between invertebrates and vertebrates establishing this particular organism as an excellent genetic model. Because of its small size, large populations and self-fertilization of the hermaphrodite, functional predictions carried out by genetic modifications as well as RNAi screens, can be rapidly tested. This is an obvious advantage when compared to the increasingly complex
Drosophila and mouse genomes. Therefore, the nematode C. elegans has emerged as an excellent entry point to begin to address these predictions. There are currently two main approaches in C. elegans to investigate gene function on a genomic scale using reverse-genetics. The first one is based on RNA-mediated interference (RNAi) where "functional knock-outs" of a particular gene can be studied and phenotypes identified. The other is a PCR -based technology to identify genetic mutants in a mutagenized library. 
Complementary expression data can also be acquired from microarray data or by Serial Analysis of Gene Expression (SAGE) to identify genes that are co-expressed or up/ down regulated under defined conditions. These methods can be directly combined, on a particular set of genes, to provide a comprehensive description of the gene's expression patterns and functions [1]. In addition, results can be refined using independent screens of other experimental systems, for example human cell lines.

RNAi based on the introduction of double-stranded RNA (dsRNA) is the method that results in specific inactivation of the corresponding gene through the degradation of endogenous mRNA. It was originally described in C. elegans $[2,3]$ and has become the main reverse-genetics tool for determining the function of specific genes. Several large-scale studies involving C. elegans [4-7], subjected approximately $90 \%$ of the 19,427 predicted genes to down regulation via RNAi. Moreover, individual clones of the entire RNAi feeding library described in Fraser et al. [5] can be ordered directly. In addition, in vitro synthesis of 21-nt small interfering RNAs to mediate gene-specific suppression in mammalian cells have been developed in order to extend this particular technique to higher eukaryotes [8].

$80 \%$ of C. elegans genes have human homologs [9]. As long as we are able to establish orthology, information obtained for a gene sequence in one organism is potentially transferable to the other [10]. Nevertheless, since the human and the worm genomes are phylogenetically very distant, in many cases these sequences are only able to produce poorly supported trees. Moreover, position of individual branches may not be conserved between different algorithms for finding distance trees. Phylogenetic reconstructions involving human/worm sequences in a number of cases are unable to resolve their exact phylogenetic past. In the case of orthology assignments they provide only an indication for its existence.

Moreover, sequence orthology does not necessarily imply the same function. However, genes shown to be descendants of the same gene (orthologous genes) have, in general retained the same function over the course of evolution [10]. If so, it is important to select for analysis only the genes where a clear orthology assignment can be established.

Based on a collection of uncharacterized protein families derived from a comparison of three available genomes including $H$. sapiens, C. elegans and D. melanogaster, an integrated in-house program for functional gene annotation was initiated. As part of this annotation effort, a set of genes originating from this collection of novel protein families was selected for functional analysis in both C. elegans and human cell lines.

In this study novel protein families are explored using a diverse set of technologies involving both in silico and experimental analysis with the intention of identifying interesting gene candidates showing evidence of highly conserved function which may serve as potential drug targets in the future.

We have sought to identify, through phylogenetic analysis, the most likely worm orthologs for a set of human genes. Expression patterns and RNAi data in the worm were obtained, as well as corresponding data using human cell lines.

\section{Results and Discussion \\ Phylogenetic analysis: the most likely ortholog?}

Multiple gene duplication followed by massive gene loss and acquisition of novel functions has been shaping the evolution of distant organisms. In a search for the most likely orthologs, special attention must be paid to the fate of duplicated genes when related to speciation. Duplicated genes tend to evolve in different patterns following the duplication event arising from different functional constrains [11].

According to Remm et al. and Sonnhammer and Koonin, out-paralogs are paralogs that predate speciation $[12,13]$. By contrast, in-paralogs are genes that arose after speciation. Therefore, all in-paralogs are considered potential functional orthologs. Using translated sequence we compare maximum parsimony trees to neighbor joining counterparts. Genes for which the position of the branches (in particular the branches leading to C. elegans and its closest human homolog) was conserved between both algorithms chosen for finding distance trees (Neighbor joining versus Maximum Parsimony), were selected for the experimental study (Tab. 1.). This criterion has been used in order to eliminate genes producing unstable topologies caused by duplications or a generally weak phylogenetic signal. Also, in this way it was possible to infer the homolog which most likely retained the ancestral gene function.

Unique orthologous gene pairs are difficult to identify using standard similarity searches, as multiple candidate genes are typically obtained. Our trees typically contain two or more human homologs showing significant sequence similarity to a single worm gene (Fig. 1). Therefore, we selected candidate genes by choosing genes that produced trees that allow for identification of single human sequences as the most probable orthologs. These trees clearly discriminate out-paralogs or other in-paralogs (Fig. 1A and 1B). In effect, well-supported trees 
Table I: Wormbase ID numbers and accession numbers for the analyzed set of $C$. elegans genes and their identified human homologs.

\begin{tabular}{llll}
\hline C. elegans & & Human ortholg & \\
\hline Worm base ID & Description & Acession no. & Description \\
\hline T24DI.I & Chondroitin synthase & NP_055733 & Chondroitin synthase I \\
F23C8.6 & Predicted coding sequence & NP_065I45 & CHMPI.5 protein \\
F38H4.7 & Predicted coding sequence & AAK25825 & BTB/POZ domain containing protein I \\
C05C8.6 & Predicted coding sequence & NP_060267 & BTBD2 protein \\
C0IA2.4 & Predicted coding sequence & NP_054762 & Hypothetical protein \\
CIID2.4 & Predicted coding sequence & NP_II5683 & C9orf64 protein \\
F4ID9.1 & Predicted coding sequence & NP_056520 & Hypothetical protein \\
C47DI2.2 & Predicted coding sequence & NP_060I23 & Dyggve-Melchior-Clausen syndrome protein \\
ZK795.3 & Predicted coding sequence & NP_219484 & U3 snoRNP protein 4 homolog \\
CI7E4.3 & Predicted coding sequence & NP_848545 & Hypothetical protein MGC48332 \\
B0379.4a & Predicted coding sequence & AAP34400 & HYA22 protein. May be tumor supressor \\
C09D4.I & Predicted coding sequence & NP_06026I & Feline leukemia virus subgroup C receptor-related protein 2 \\
Y45FI0A.6a & Predicted coding sequence & BAA74905 & Hypothetical protein KIAA0882 \\
& & & \\
\hline
\end{tabular}

showing a one-to-one relationship between human and worm sequence were selected. However, since the purpose of this analysis was to identify genes that most likely retained the same function rather than orthology sensu stricto, it is possible that in some cases a paralog (in-para$\log$ ) was selected as the most promising ortholog. In other words, the C. elegans genes that we identified are either true orthologs or the best in-paralogs to the corresponding human genes [12]. Despite the fact that the exact type of orthology encountered in individual trees could not always be identified, we find this level of resolution satisfactory for the purposes of this study.

Thus, phylogenetic analysis (Fig. 1A and 1B) predicts F41D9.1 (Q94222) to have a similar function to human CAA18266. The tree also assigns other human sequences, namely BAA83007 and AAL55877, which are apparently a product of a duplication event in the mouse/human lineages, as either out-paralogs or in-paralogs. Regardless of their exact orthology/paralogy relation to the worm sequence, neither of them is the most probable ortholog.

To the contrary, a frequently encountered situation was obtained in the case of the worm gene C13F10.4 (Fig. 1C and 1D). The two human sequences though rather similar having a protein matrix distance of 0.61482 (Philip package), were easily separated in both the Neighbor Joining tree (NJ tree) and the Maximum Parsimony tree (MP tree). The NJ tree in particular demonstrates that the gene was duplicated in the animal series starting from Gallus sp. and upwards. Thus, in an ideal case, phylogenetic analyzes would correctly identify the exact copy of the duplicated gene in the human genome and assign it to a single C. elegans sequence as its true ortholog, as shown for F41D9.1
(Fig. 1A and 1B). The position of the other copy would point to its paralogous/in-paralogous relation to the worm sequence. However, the worm gene is either weakly associated with the branch leading to both human sequences (NJ tree, Fig. 1C) and it forms a separate branch with the XP_113763 human sequence (MP tree, Fig. 1D).

C. elegans genes and their most probable human orthologs are shown in the Tab. 1. Interestingly, due to the strict criteria we applied in order to select the most likely human orthologs, even C27A7.1, a known disease gene http://www.wormbase.org, which is regarded as orthologous to the human gene NPPASE (ENPP1; OMIM:173335) was not identified.

\section{Expression profiles and RNAi phenotypes}

A distinct GFP expression: F4ID9.I, CI 7E4.3, ZK795.3 and CO9D4.I

Here we report GFP expression under the control of a putative promoter for F41D9.1 that occurs predominantly in many neural cells in the head around the posterior pharyngeal bulb, along the ventral nerve cord and in the tail (Fig 2). Head neurons include clusters in the dorsal and retrovesicular ganglia as well as the sensory amphid neurons. Expression is present in both larval and adult stages. No other phenotype was observed following down-regulation which is in accordance to previously published results by [14].

The C17E4.3::GFP reporter is expressed in the developing embryo and L1 larval stages, in three distinct sheath/ socket cells in the head region close to the anterior bulb of the pharynx and several cells around the anus (Fig 3). Expression is also seen in several pharyngeal muscle cells. 


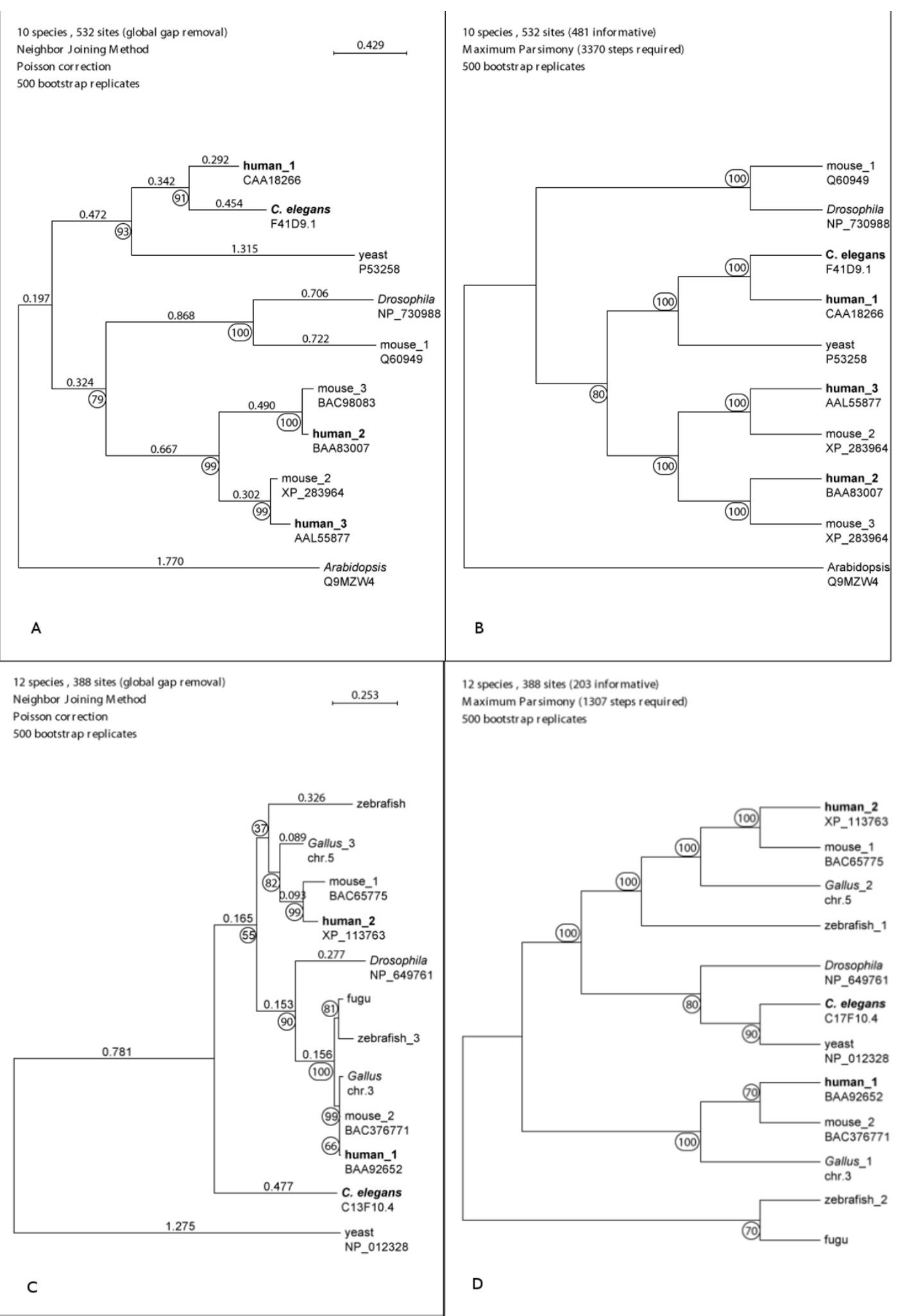

Figure I

Maximum parsimony and Neighbor joining trees for F4ID9.I (A, B) and CI3FIO.4 genes (C,D). Illustration of the criteria that has been applied in order to select the genes. Only genes able to produce trees as shown in A and B (F4ID9.I) were subjected to experimental work. 

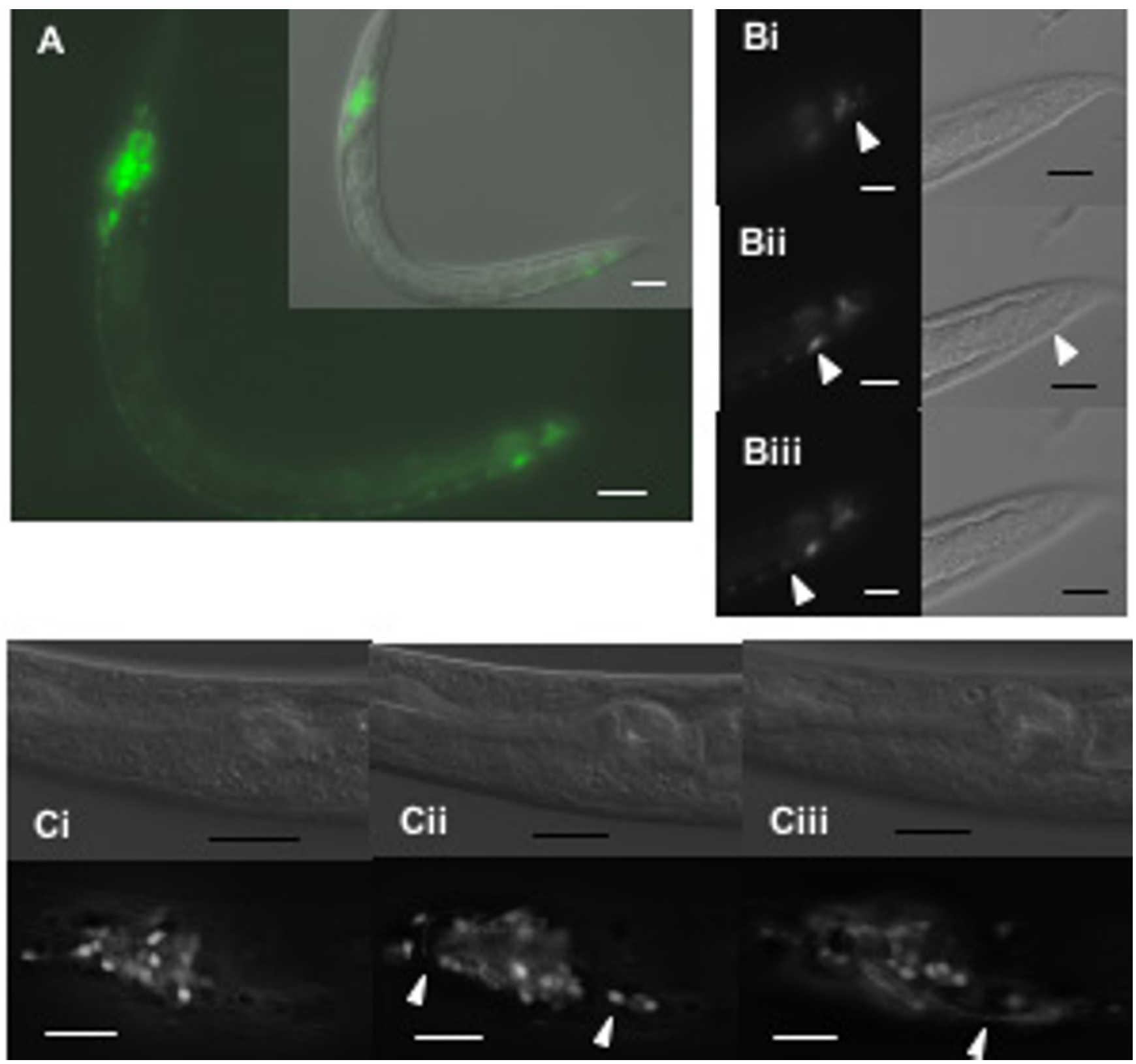

Figure 2

F4ID9.I::GFP expression. Widespread through the neural system. Panel A shows the general expression pattern in an LI stage animal. Many cells in the nerve ring, the ventral nerve cord (vnc) and the tail region express GFP. Panel B presents the tail region in greater detail, scanning through the animal at three focal planes from right (Bi) to left (Biii), with the ventral side facing down. A cluster of laterally symmetrical cells is visible in panels $\mathrm{Bi}$ and Biii, whereas in Bii cells of the vnc are visible. Magnification is 100x. Fig $C$ presents 3 focal planes from dorsal (Panel $\mathrm{Ci}$ ) to ventral (Panel Ciii) through the worm head, with the posterior pharyngeal bulb to the left. The GFP images have undergone deconvolution to increase resolution. Cells of the dorsal ganglion are visible in $\mathrm{Ci}$, the retrovesicular ganglion is marked in $\mathrm{Cii}$, and processes leading to it are indicated in Ciii. Scale bar represents $10 \mu \mathrm{m}$. 

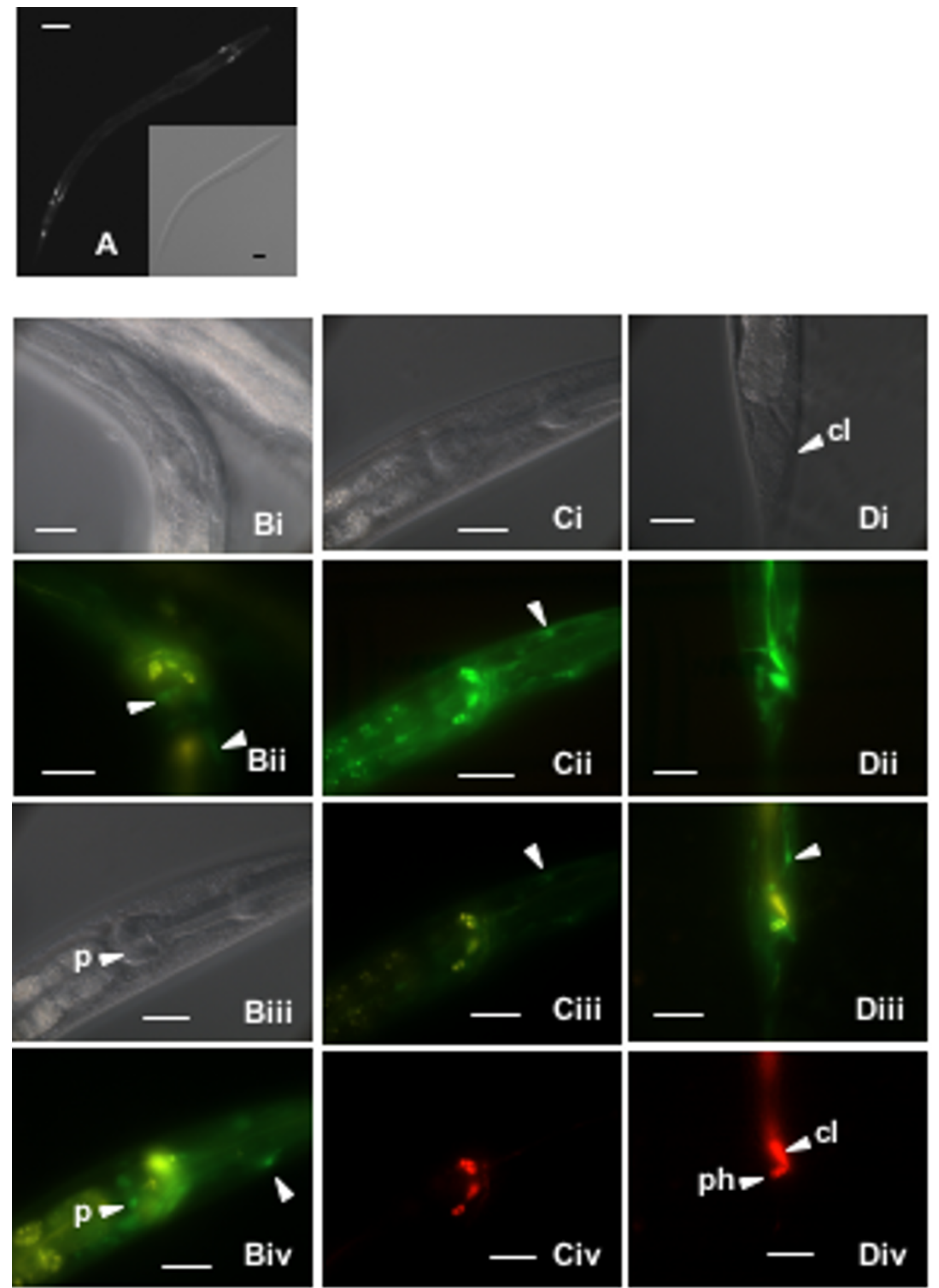

Figure 3

CI 7E4.3::GFP reporter expression. Present in the head and tail regions of larval stages. Panel Ai shows an overview of an LI animal, with distinct cells near the anterior pharyngeal bulb as well as in the tail. Close up of the head reveals GFP expressing cells (Panel Bii), including muscle cells located in the posterior pharyngeal bulb (Panel Biv, labeled 'p'). Sheath/socket cells located at the anterior pharyngeal bulb are indicated in panels Biv, Dii and Diii. Dye-filling tests (see Materials and Methods) to stain sensory amphid (head) and phasmid (tail) neurons are shown in panels $C$ and $D$. The amphids are specifically visualized in panel Civ (red fluorescence), and under an FITC filter in Ciii (yellow fluorescence, not in nucleus) and green with an EGFP filter, and are not the same as the cells expressing the CI7E4.3::GFP reporter (arrow in panels Cii and Ciii). In the tail, phasmids (labeled 'ph') are clearly seen in panel Div just below the anus (cl), but are not expressing GFP, which is instead present in several hypodermal cells (arrow panel Diii). Scale bar represent $10 \mu \mathrm{m}$. 

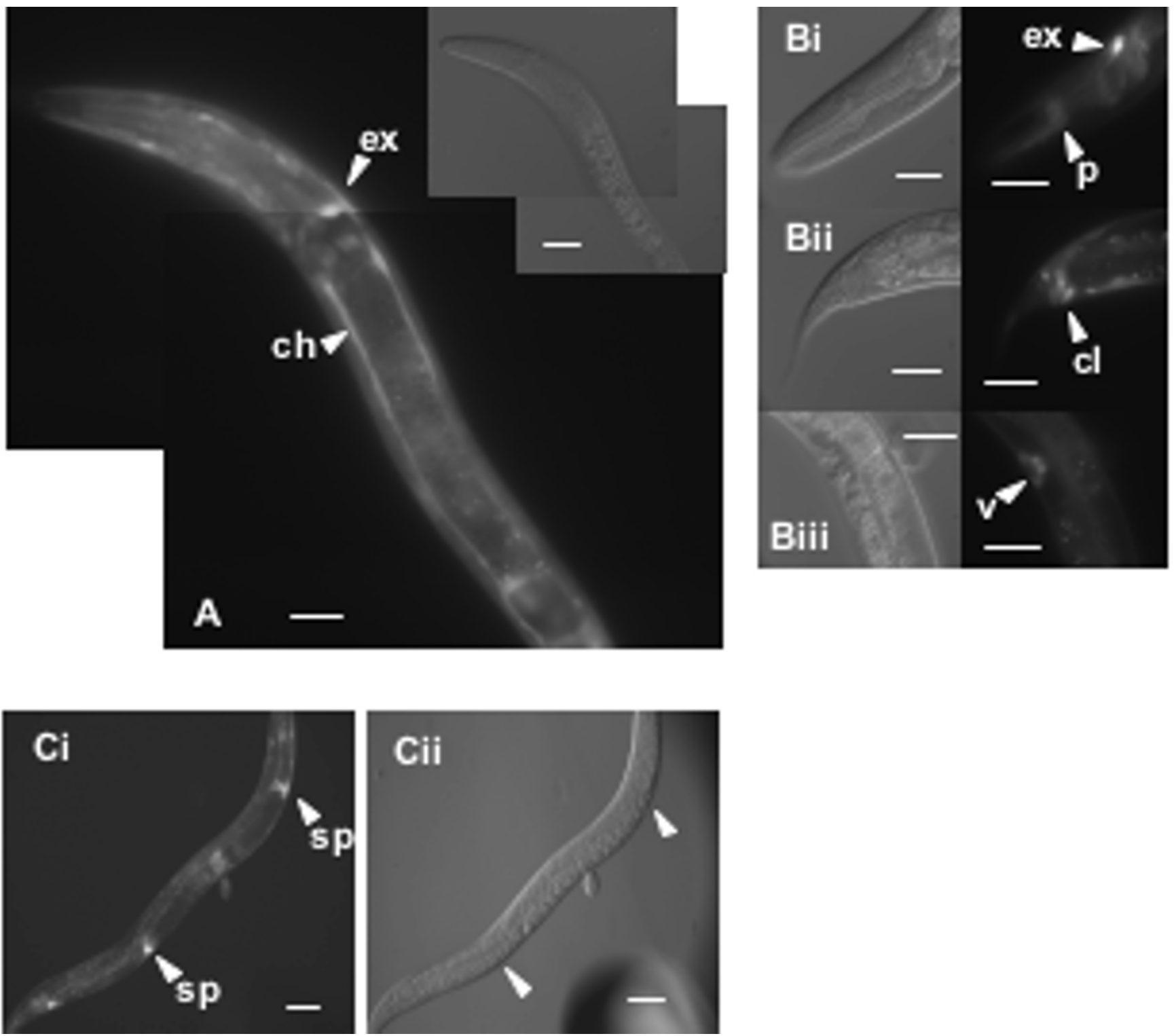

\section{Figure 4}

ZK795.3::GFP reporter expression. Evident in the excretory cell system (panel A) in about $20 \%$ of animals, but was always present in the excretory cell (labeled 'ex', panels A and Bi). The anal sphincter and/or depressor cell around the anus ('cl') also expressed GFP (panel Bii), as did the juvenile vulva ('v', panel Biii) and the spermathecae (panel C). Scale bar represents $10 \mu \mathrm{m}$.

Dye filling tests confirmed that the processes extending to the nose were not from amphid neurons, but rather from socket cells (Fig 3, panel C). The expression occurs exclusively in the developing embryo and L1 larval stage suggesting involvement of the gene in development. No RNAi phenotype was associated with this gene. The wild type phenotype, following down-regulation, was also obtained by Simmer et al. [7]. However, the results obtained by Piano et al. [15] show that silencing of the gene results in embryonic lethality. Nevertheless, inconsistent phenotypes have been obtained by different groups for several other genes [16].

ZK795.3::GFP expression pattern includes spermatheca, hypodermal cells, pharynx and the excretory cell and channels (Fig 4). In the L3 stage, expression was seen in the vulva, and in P6.p descendants. 

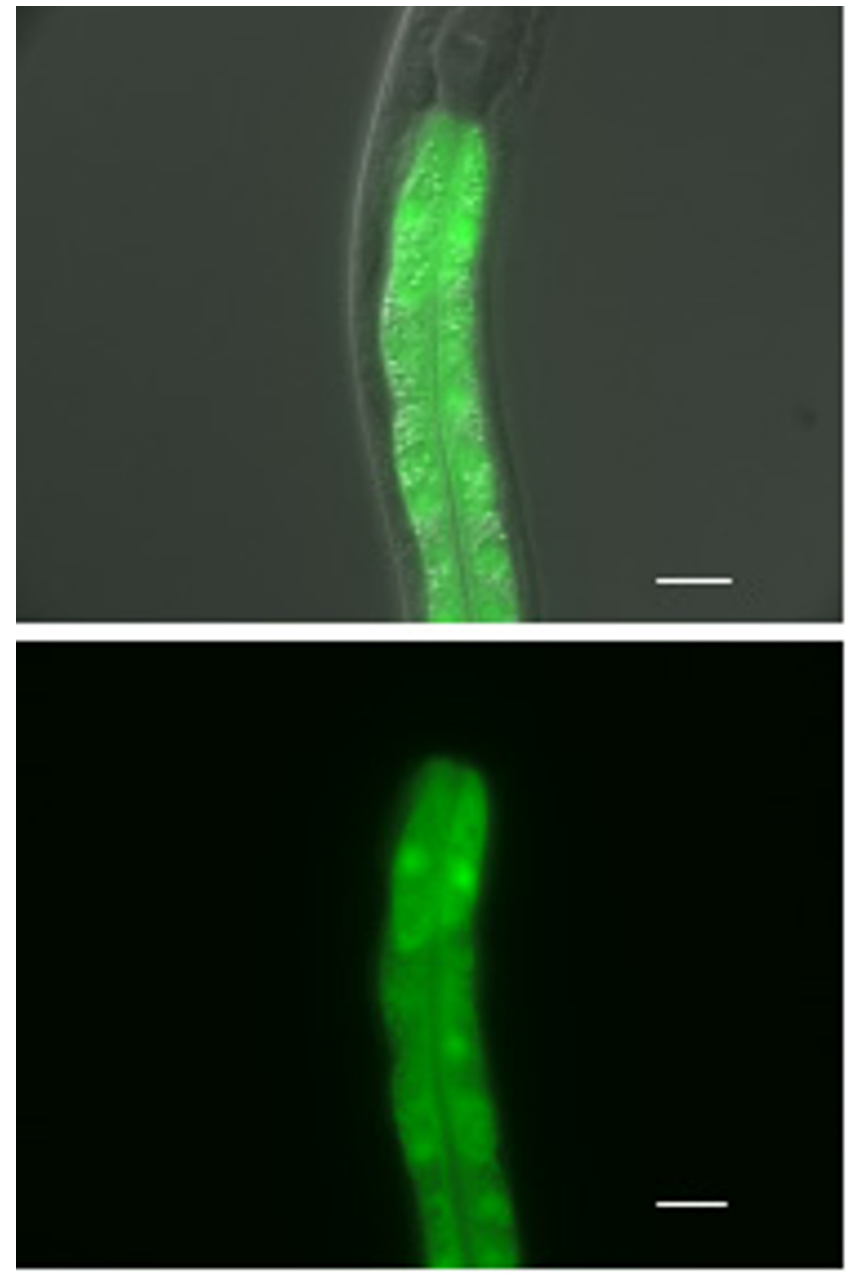

Figure 5

C09D4.I::GFP expression. Limited to the nuclei of intestinal cells. The top figure is an overlay of the DIC and GFP images. Scale bar represents $10 \mu \mathrm{m}$.

C09D4.1 had a simple expression pattern limited to intestinal cells in all developmental stages (Fig 5).

Visible RNAi phenotypes: T24D I.I, F23C8.6, ZK795.3 and B0379.4a

To investigate in detail the RNAi effects on growth, brood size and life span, we have closely followed a population of embryos (N2 and rrf-3 (NL2099) to full development at $20^{\circ} \mathrm{C}$, in RNAi plates for corresponding genes with visible RNAi phenotypes.

C. elegans SQV-5 14162 protein (WP:CE 14162) encoded by T24D.1 gene is a chondroitin synthase that initiates/ elongates chondroitin chains. This protein is also required for cytokinesis, gonad migration and vulval morphogene- sis where it possibly promotes filling an extracellular space with fluid [17].

Following exposure to the corresponding dsRNA, a smaller F1 population size was observed when compared to the control animals in the both N2 and NL2099. F2 animals show a robust RNAi-induced phenotype including: sickly appearance, partial sterility, and small brood size. Lethality was observed in F3 embryos in the rrf-3 background at $20^{\circ} \mathrm{C}$. The partial sterility among the F2 animals is in accordance with the reported http:// www.wormbase.org mutant phenotype (self-sterile hermaphrodites). All the phenotypes indicated above were reduced in the $\mathrm{N} 2$ background and also when grown at $15^{\circ} \mathrm{C}$. Additional mutant phenotypes included squashed vulva (Sqv), and reduced L4 vulva invagination http:// www.wormbase.org. However, according to Fraser et al. [5], down-regulation via RNAi did not produce a distinct phenotype. This is possibly due to the large-scale nature of their study and its focus on early developmental stages.

For F23C8.6, our results revealed slow growth (Gro) and uncoordinated behavior (Unc) which are both in agreement with the results obtained by Fraser et al. [5]. Our observations also confirmed larval arrest as reported previously [7]. As with T24D.1, ZK795.3 and B0379.4a, down-regulation of F23C8.6 did not affect life span. Gene function related to growth, larval development and locomotory behavior have been also inferred from the mutant phenotype http://www.wormbase.org.

Down-regulation of ZK795.3 affects the growth rate significantly in both wild type and the hypersensitive NL2099 strain (rrf-3). At $20^{\circ} \mathrm{C}$ growth was $10-15$ times slower then the wild type control, slightly more pronounced in the rrf-3 strain. A large proportion of sterile F2 (Stp) animals was also observed. The minority of animals that were fertile produced very few eggs but these eggs were as viable as wild type. According to the previous studies down-regulation of the same gene has produced the following phenotypes: Gro, Emb, Stp, Lva $[7,14,15]$.

Our results for down-regulation of B0379.4a are consistent with the data obtained by Kamath et al. [14]. RNAi resulted in an Egl phenotype that was more pronounced in the $r r f-3$ background. The involvement of the gene in oviposition was inferred from the mutant phenotype http://www.wormbase.org. The gene has orthologous sequences in both the human and mouse genomes that code for a small CTD phosphatase and nuclear LIM interactor-interacting factor 2 , respectively.

RNAi on C05C8.6 has been reported by Simmer et al. as Emb, Lva and Lvl [7]. However, our results are consistent with another study published by Kamath et al. where no 
specific phenotype was observed [14]. The mutant phenotype of C05C8.6 points to its involvement in embryonic and larval development with a molecular function associated with protein binding http://www.worm base.org.

Although a greater proportion of genes show specific RNAi phenotypes when using rrf-3 strain [7], our results point to an increase in the severity of the phenotypes following down-regulation in the rrf-3 background, rather than additional phenotypes.

\section{Subcellular localization in human cell lines}

For seven of the human orthologs presented in this study, we cloned full-length open reading frames (ORFs), representing encoded cDNAs, into vectors containing V5 epitope sequences in order to generate recombinant fusion proteins for immunofluorescence detection. Because of the novelty of these proteins and the lack of available antibodies against such proteins, it was necessary to overexpress in human cell lines and subsequently detect with antibodies against the V5 fusion tag. Subcellular localizations were obtained in two human cell lines: HeLa and Human Embryonic Kidney 293 (HEK293). Endogenous expression for the proteins studied in the two cell lines was confirmed by RT-PCR.

NP_219484, human ortholog for ZK795.3, displays three distinct patterns: diffuse nuclear, nucleolar and nuclear foci (Figure 6A). We were able to confirm the nucleolar pattern by colocalization with fibrillarin, a protein predominantly found in nucleoli and cajal bodies. We were unable to determine the exact sub-compartment to which the nuclear foci belong. However, the pattern is strikingly similar to that of paraspeckles. The proteins, described as U3 snoRNP protein 4 homologs, belong to the IMP4 family of proteins which are small ribonucleoproteins involved in pre-ribosomal RNA processing. These results are consistent with those previously obtained in yeast and recently described in human [18]. Based on the interaction of these proteins with snoRNA in 60-80S RNP complexes and their likely involvement in pre-rRNA processing, it is possible to speculate that the preferred location of the Imp4 protein is both transient and dynamic throughout the nucleus, which would explain the three patterns we observe.

NP_848545 also described as hypothetical protein MGC48332 and predicted ortholog of worm protein C17E4.3 displayed a nuclear and perinuclear pattern covering the entire circumference of the nucleus but did not appear to be inside the nucleus. We were able to confirm the presence of this protein at the nuclear membrane by colocalization with Lamin, denoting a possible role for this protein as part of the nuclear envelope (Figure 6B).
C3HC4 which contains a RING-finger motif, has been classified as the third member (MARCH-III) of the recently defined membrane-associated RING-CH protein family [19]. The same authors reported that proteins belonging to this family, including MARCH-III, characteristically contain two predicted C-terminal transmembrane domains, indicating a possible association with membrane-bound organelles. Furthermore, Bartee et al. concluded that localization of human MARCH III by fusion protein overexpression revealed a punctate pattern partially overlapping with cytoplasmic vesicles, specifically early endosomes. However, we were unable to confirm this finding. It is important to note that proteins are, in many cases, dynamic in location meaning that there are potentially multiple locations. Thus, our co-staining data are the most compelling we have seen.

AAK25825/NP_079514, also described as BTB/POZ domain containing 1 (BTBD1), exhibited a cytoplasmic pattern resembling elongated "worm-like" bodies for which we were unable to determine a known structure (data not shown). Members of this domain family have been shown to interact with co-repressor complexes involved in transcriptional repression [20-22]. Previously, BTBD1 has been shown to interact specifically with topoisomerase I [23]. In addition, a recent study further characterized BTBD1 as colocalizing with TRIM family members [24]. Interestingly, TRIM proteins have been shown to exhibit ubiquitin ligase activity.

NP_115683 has no functional annotation and has only been described as an open reading frame located on human chromosome 9. This protein exhibits weak expression when "overexpressed" under control of CMV promoter. Immunofluourescence shows this protein to have both a cytoplasmic and a nuclear distribution. Furthermore, the cytoplasmic expression appears punctate and partially colocalizes with calreticulin, a marker for endoplasmic reticulum (Figure 6C).

AAP34400.1/JC5707 is a member of a family of small Cterminal domain phosphatase (SCP3) and contains an NIF domain (Nuclear Lim Interacting factor-like phosphatase). Other members of this family have been shown to interact with RNA polymerase II and show nuclear localization (SCP1) [25]. Surprisingly, SCP3 was detected at the plasma membrane by colocalization with annexin II, a known component of the plasma membrane (left panel, Figure 3D). In addition to this pattern, we noted fibrous structures that partially colocalized with beta-actin (right panel, Figure 6D).

Hypothetical protein NP_056520 expression was detected by western blot and immunofluorescence microscopy (data not shown). Microscopy detection displayed a 

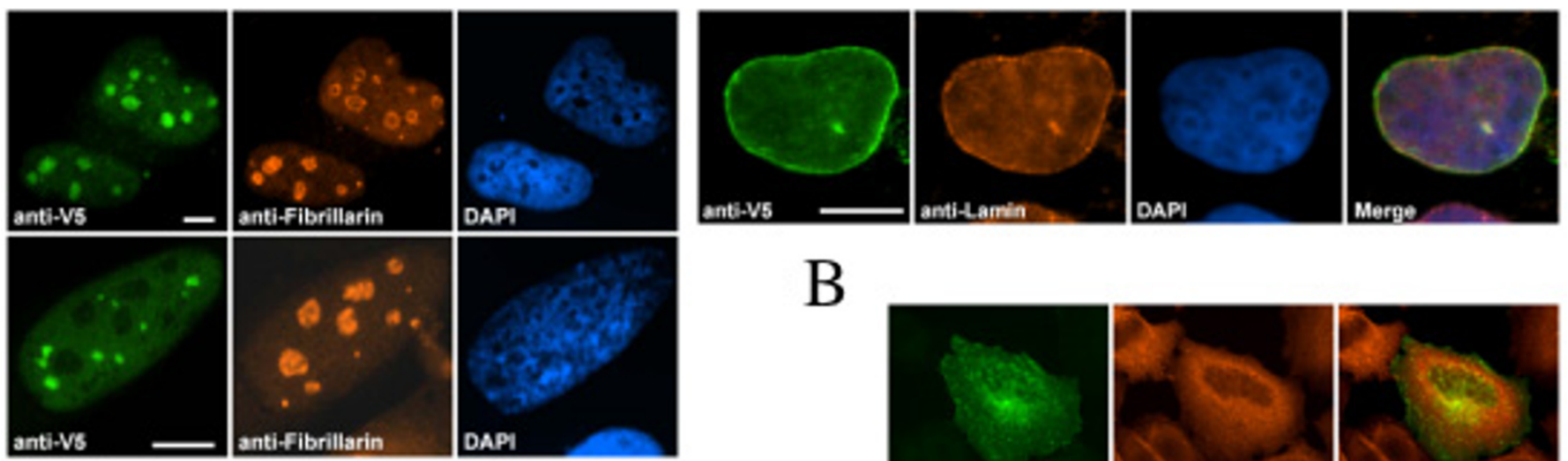

A
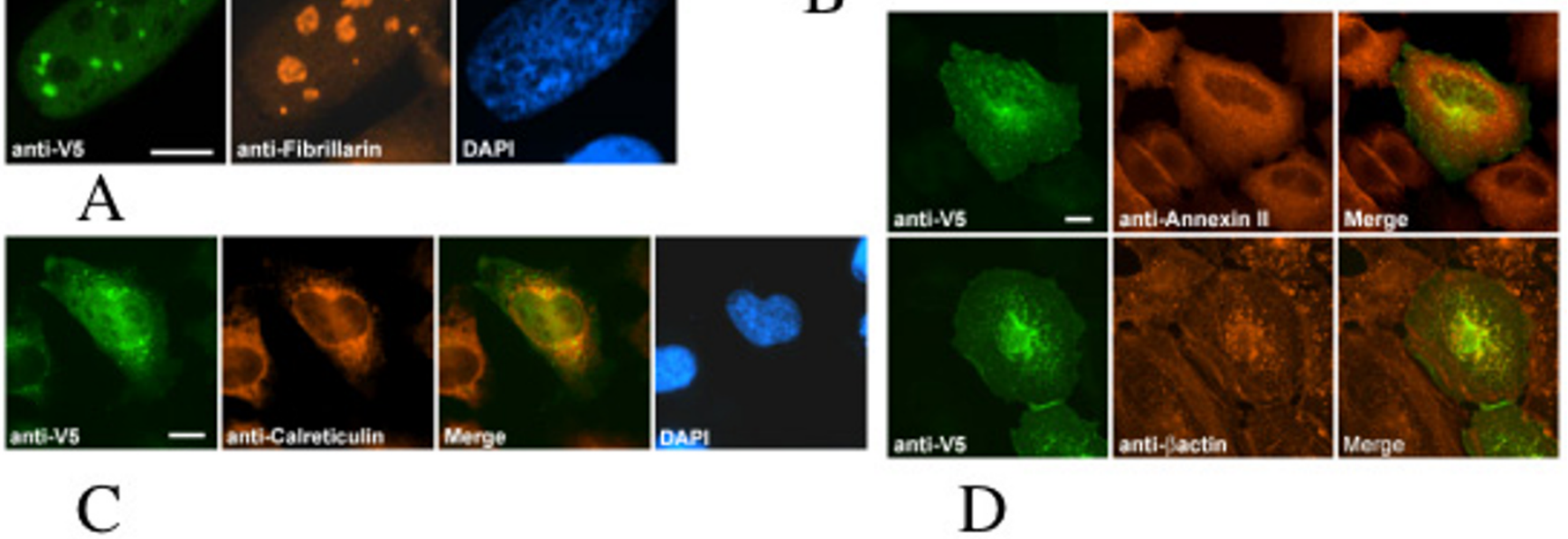

D

Figure 6

Subcellular localization of recombinant fusion proteins in human cell lines. Human protein NP_219484 (C. elegans protein ZK795.3) was detected in transfected HeLa cells with goat anti-V5 conjugated with FITC and co-localized with human anti-fibrillarin subsequently detected by donkey anti-rabbit Cy3 (A). Nuclei were stained with DAPI. This protein displays both a nucleolar (top row) and nuclear speckle (bottom row) pattern (A). NP_848545 (C. elegans protein CI7E4.3) was detected in HEK293 cells with mouse anti-V5 and goat anti-mouse Alexa 488 (B). Cells were costained with rabbit anti-Lamin and donkey anti-rabbit Cy3 illustrating a colocalization with the nuclear envelope (B). Partial ER and nuclear distributions were observed for NP_I I5683 by co-detection with rabbit anti-calreticulin in HeLa cells (C). AAP34400.I was detected in HeLa cells at the plasma membrane by co-staining with Annexin II (left panel, D) and a partial colocalization with beta-actin was also detected (right panel, D). Scale bars represent $10 \mu \mathrm{m}$.

cytoplasmic/nuclear rim staining pattern. However, western blot analysis revealed a molecular weight of approximately $50 \mathrm{kDa}$ which is inconsistent with the predicted size of $80 \mathrm{kDa}$, indicating a possible premature stop in translation or post-translational cleavage. In addition, we were unable to detect ectopic expression for NP_060123. Any attempts to detect this protein via western blot or microscopy were unsuccessful despite sequence validation of the corresponding expression plasmid.

\section{Conclusion}

Specific expression patterns have been identified for the F41D9.1, ZK795.3, C17E4.3 and C09D4.1 GFP-fusion strains. Visible RNAi phenotypes for T24D1.1, F23C8.6, ZK795.3 and B0379.4a were observed. RNAi on T24D1.1a produced an extensive phenotype that was not revealed in the large-scale study by Fraser et al. [5]. Thus, our study shows the value of analyzes focusing on a small number of candidate genes where a variety rather then a predefined set of phenotypes are observed. In addition, immunofluorescence microscopy of human cell lines over-expressing NP_219484, NP_848545, AAK25825/ NP_079514, NP_115683, AAP34400.1/JC5707 and NP_056520 has detected subcellular localization of the corresponding proteins. The most complete data set comprising both a visible RNAi phenotype or/and a distinct GFP expression in C. elegans and a subcellular protein over-expression profile using human cell lines has been obtained for ZK795.3 (NP_219484), F41D9.1 (NP_056520) and C17E4.3 (NP_848515) (Tab. 2).

In the case of genes F41D9.1 and C17E4.3, a mainly neuronal GFP expression was detected. Therefore, the absence of an observable RNAi phenotype may be explained by the known refractory nature of RNAi in this tissue. For the gene ZK795.3, which showed a more generalized effect on 
Table 2: The genes for which a visible RNAi phenotype or/and a distinct GFP expression in C. elegans and a subcellular protein overexpression profile using human cell lines has been obtained.

\begin{tabular}{|c|c|c|c|}
\hline $\begin{array}{l}\text { Worm gene / Human } \\
\text { ortholog }\end{array}$ & RNAi (C. elegans) & GFP expression (C. elegans) & Subcellular localization (human) \\
\hline T24DI.I / NP_055733 & $\begin{array}{l}\text { Smaller population size, sickly } \\
\text { appearance, partial sterility, smaller } \\
\text { brood size }\end{array}$ & No specific expression observed & Cloning failed \\
\hline F23C8.6 / NP_065I45 & $\begin{array}{l}\text { Slow growth, uncoordinated } \\
\text { behavior, larval arrest }\end{array}$ & No specific expression observed & Cloning failed \\
\hline F38H4.7 / AAK25825 & Wild type & No specific expression observed & Cytoplasmic foci \\
\hline CIID2.4 / NP_II 5683 & Wild type & No specific expression observed & $\begin{array}{l}\text { Weak cytoplasmic and nuclear } \\
\text { pattern }\end{array}$ \\
\hline F4ID9.I / NP_056520 & Wild type & $\begin{array}{l}\text { neural cells in the head, along the } \\
\text { neural nerve cord and in the tail }\end{array}$ & Cytoplasimic/nuclear rim \\
\hline ZK795.3 / NP_219484 & Slower growth, sterility, & $\begin{array}{l}\text { Spermatheca, hypodermal cells, } \\
\text { pharynx, excretory cell and channels }\end{array}$ & $\begin{array}{l}\text { Diffuse nuclear, nucleolar, nuclear } \\
\text { foci }\end{array}$ \\
\hline CI7E4.3 / NP_848545 & Wild type & $\begin{array}{l}\text { Three distinct sheath/socket cells in } \\
\text { the head; several cells around the } \\
\text { cloaca; pharyngeal muscle cells }\end{array}$ & Perinuclear/nuclear lamina \\
\hline B0379.4a / AAP34400 & Egg-laying deffects & No specific expression observed & Cytoskeletal/plasma membrane \\
\hline C09D4.I /NP_06026I & Wild type & Intestinal cells & Protein not detected \\
\hline
\end{tabular}

the development, corresponding widespread expression pattern was observed in the worm and in human cell lines. Given that the protein prediction indicates this gene as a snoRNP candidate, its involvement in a variety of cellular processes thus can be expected. To the contrary, restricted subcellular expression patterns in human cells observed in a few cases, for example the AAK25825 (F38H4.7) and NP_115683C11D2.4 genes (Tab. 2), correlate with the absence of phenotypes other than the wild type. This fact suggests that these genes may be of somewhat lesser importance.

In conclusion, this comparative C. elegans-human cell lines study based on orthology assignments explores gene functionality by combining two key aspects of functional genomics: a whole organism approach and protein overexpression data using cells lines.

Our results extend currently available information on the selected genes providing a step more towards identifying their exact function.

\section{Methods \\ Phylogenetic analysis}

In order to select candidate genes for the RNAi experiments phylogenetic reconstructions were performed as follows: C. elegans and related sequences were collected from Genelynx http://www.genelynx.org or/and Wormbase http://www.wormbase.org. The data set typically included several homologs of the model organisms (yeast, fly, etc.) and one or more human sequences. Multiple sequences alignments were done using ClustalW [26]. Phylogenetic trees implemented by the SeaView and
Phylo_win program [27] were constructed by using maximum parsimony and the neighbor-joining method with 500 bootstrap replicates. Only genes producing well-supported trees (bootstrap value $>50$ ), with the same branch positions, when applying both maximum parsimony and neighbor joining method were selected (Fig. 1.).

\section{Generation of bacterial feeding library}

Total N2 RNA extract was used to synthesize cDNA (Reverse Transcription System; Promega). A pair of oligonucleotides that had restriction sites for XmaI (extremely rare sites in the worm genome) was designed for each predicted coding sequence. PCR products of the selected genes were generated using the RT-PCR mixture as template. In addition, primers for spliced leaders SL1 and SL2 were also used. PCR products were ligated into previously digested L4440 (double-T7 vector, Fire lab, http:// genome-www.stanford.edu/group/fire/) using Rapid DNA Ligation Kit (Roche). Plasmids were transformed into E. coli JM109. Plates were screened for recombinant clones by restriction analysis of plasmid minipreps. Positive clones were grown in overnight cultures. Plasmids were extracted by QIAfilter Plasmid Kit (QIAGEN). Clones were sequenced and used to transform E. coli HT115, an RNaseIII-deficient strain used to feed the nematodes.

\section{RNAi by feeding}

RNAi plates were prepared by spreading $200 \mu \mathrm{l}$ of the bacterial liquid cultures per small $(6 \mathrm{~cm}$ diameter $)$ NGM plates supplemented with $1 \mathrm{mM}$ IPTG and $25 \mu \mathrm{g} / \mathrm{ml}$ carbenicillin. Plates were seeded with N2 eggs prepared with the standard bleaching method. The hatched worms 
and their progeny (four generations) were screened for phenotypes other than wild type. As a positive control, the HT115 E. coli strain transformed with the unc-22 gene ("twitchin") was used (Fire lab vector pPD34.09). N2 strain was incubated at 15 and $25^{\circ} \mathrm{C}$. All the experiments were repeated using the NL2099 strain (rrf-3) incubated at 15 and $20^{\circ} \mathrm{C}$ since it is known for a temperature-dependent decrease in the brood size $[28,29]$. Plates seeded with the empty vector were used as a negative control.

\section{Gene expression profiles}

GFP strains were made by transcriptional fusions of putative promoters $(1-2.5 \mathrm{~Kb})$ with GFP using a fusion PCR technique as described by Hubert O. [29].

\section{Estimation of the life span, brood size, embryonic lethality and duration of the larval stages}

NL2099 and N2 worms were grown on plates seeded with bacteria containing double-T7 (L4440) vector without insert and as well as corresponding RNAi plates. Individual $F_{1}$ eggs were transferred onto 10 fresh plates and their development followed at $20^{\circ} \mathrm{C}$. Individual worms were transferred every $24 \mathrm{~h}$ onto fresh plates. The total number of $\mathrm{F}_{2}$ eggs on the plate was counted, as well as the number of eggs that did not hatch. Individual worms were observed daily until they died to ascertain their lifespan.

\section{Microscopy}

Microscopy was carried out with a Zeiss Axioplan system complete with filters for visualizing rhodamine and GFP fluorescence. A Hamamatsu black and white and Zeiss Axiocam colour camera were used for capturing images. Images were processed in Adobe Photoshop. Some images were processed with Openlab 3D restoration software to increase image clarity.

Dye filling experiments were done to assist in identifying cells. Briefly, worms were incubated for an hour in DiI stain and observed with a microscope under UV light through a rhodamine filter. Only amphids and phasmids were stained.

\section{Exogenous protein expression and immunofluorescence detection in human cell lines}

Plasmid constructs containing full-length genes of interest were generated according to previously described methods [30]. Briefly, full-length open reading frames were cloned into a mammalian expression vector containing a CMV promoter and an in-frame $\mathrm{N}$-terminal or C-terminal V5 epitope tag in order to generate fusion proteins for expression studies (pcDNA-DEST40 $0^{\mathrm{TM}}$, Invitrogen). HEK293 and HeLa cells (ATCC) were maintained at $37^{\circ} \mathrm{C}$, $5 \% \mathrm{CO}_{2}$ in Dulbecco's Modified Eagle Medium (DMEM) supplemented with 10\% FBS and $100 \mathrm{U} / \mathrm{ml}$ Penicillin/ Streptomycin (Invitrogen). Cells were seeded on glass coverslips at $80 \%$ confluency 24 hours prior to transfection in DMEM containing 10\% FBS without antibiotics. Cells were transiently transfected for 24-48 hours with Lipofectamine2000 (Invitrogen) according to the manufacturer's recommendations. Cells were fixed with $2 \%$ paraformaldehyde/1.5\% sucrose in PBS for 15 minutes at room temperature (RT), after which cells were treated with $0.5 \%$ triton-X 100 in PBS for 5 minutes. Cells were incubated for 30 minutes at RT in blocking buffer (2\% BSA in PBS) before detection with the primary antibodies. Fusion proteins were detected with either goat anti-V5 (1:500, Bethyl Laboratories) conjugated with a FITC label or an unlabeled mouse anti-V5 (1:200, Invitrogen) for which a secondary detection with goat antimouse Alexa 488 (1:1000, Molecular Probes) was performed. Diluted antibodies were applied to coverslips and incubated at $37^{\circ} \mathrm{C}$ for 2 hours before washing in PBS. Samples were co-stained with the following organelle-specific antibodies and corresponding dilutions: rabbit anticalreticulin (1:200, Affinity Bioreagents), human anti fibrillarin (1:100, a gift from Dr. N. Ringertz, Karolinska Institute), rabbit anti Lamin (aLi, 1:200, a gift from Dr. G. Simos, EMBL), mouse anti Annexin II (1:250, BD Transduction Laboratories), mouse anti beta-actin (1:500, Sigma). For both the annexin II and the beta-actin antibodies it was necessary to perform Methanol:Acetone (1:1) fixation for 10 minutes instead of paraformaldehyde fixation and triton-x treatment.

Secondary antibody detection was performed at RT for 45 minutes with the following antibodies: anti human-Cy3 (1:1000, Amersham), donkey anti rabbit-Cy3 (1:1000, Jackson), donkey anti mouse-Cy3 (1:1000, Jackson). Coverslips were mounted on slides with Prolong Anti-Fade (Molecular Probes) mounting media. Slides were viewed by Leica DMRA2 and DMRXA microscopes with epifluorescence and images were captured with Openlab ${ }^{\mathrm{TM}}$ software version 3.1.4.

\section{Authors' contributions}

IT carried out phylogenetic analysis and experimental work of the C. elegans part, and drafted the manuscript. EH carried out protein expression and immunofluorescence detection in human cell lines. PD carried out the microscopy of the C. elegans part. RJ contributed in making transgenic strains. AVG conceived of the study and participated in its design and coordination. All authors read and approved the final manuscript.

\section{Acknowledgements}

Anna Henricson, Peter Swoboda, Erik Sonnhammer, Elisabeth Valenzuela, Jenny Redelius. Financial Sponsors: Pfizer, Genome Canada. Worms strains (N2 and NL2099 from the C. elegans center in Minnesota, vectors from the Fire lab). Robert Johnsen supported by Genome British Columbia and Genome Canada. We also want to ackowledge Thomas Bürglin for developing software specific for image processing of the C. elegans micrographs. 


\section{References}

I. McKay SJ, Johnsen R, Khattra J, Asano J, Baillie DL, Chan S, Dube N Fang L, Goszczynski B, Ha E, Halfnight E, Hollebakken R, Huang P, Hung K, Jensen V, Jones SJ, Kai H, Li D, Mah A, Marra M, McGhee J, Newbury R, Pouzyrev A, Riddle DL, Sonnhammer E, Tian H, Tu D, Tyson JR, Vatcher G, Warner A, Wong K, Zhao Z, Moerman DG: Gene expression profiling of cells, tissues, and developmental stages of the nematode C. elegans. Cold Spring Harb Symp Quant Biol 2003, 68:159-169.

2. Fire A, Xu S, Montgomery MK, Kostas SA, Driver SE, Mello CC: Potent and specific genetic interference by double-stranded RNA in Caenorhabditis elegans. Nature 1998, 391:806-8I I.

3. Montgomery $M K, X u S$, Fire $A$ : RNA as a target of doublestranded RNA-mediated genetic interference in Caenorhabditis elegans. Proc Natl Acad Sci U S A 1998, 95: I 5502-I5507.

4. Gonczy P, Echeverri C, Oegema K, Coulson A, Jones SJ, Copley RR, Duperon J, Oegema J, Brehm M, Cassin E, Hannak E, Kirkham M, Pichler S, Flohrs K, Goessen A, Leidel S, Alleaume AM, Martin C, Ozlu N, Bork $\mathrm{P}$, Hyman AA: Functional genomic analysis of cell division in C. elegans using RNAi of genes on chromosome III. Nature 2000, 408:331-336.

5. Fraser AG, Kamath RS, Zipperlen P, Martinez-Campos M, Sohrmann $M$, Ahringer J: Functional genomic analysis of $C$. elegans chromosome I by systematic RNA interference. Nature 2000, 408:325-330.

6. Maeda I, Kohara Y, Yamamoto M, Sugimoto A: Large-scale analysis of gene function in Caenorhabditis elegans by high-throughput RNAi. Curr Biol 200I, I I:I7I-I76.

7. Simmer F, Moorman C, Van Der Linden AM, Kuijk E, Van Den Berghe PV, Kamath R, Fraser AG, Ahringer J, Plasterk RH: Genome-Wide RNAi of C. elegans Using the Hypersensitive rrf-3 Strain Reveals Novel Gene Functions. PLoS Biol 2003, I:EI 2.

8. Elbashir SM, Lendeckel W, Tuschl T: RNA interference is mediated by 21 - and 22-nucleotide RNAs. Genes Dev 200I, 15:188-200.

9. Sonnhammer EL, Durbin R: Analysis of protein domain families in Caenorhabditis elegans. Genomics 1997, 46:200-216.

10. Fitch WM: Distinguishing homologous from analogous proteins. Syst Zool 1970, I9:99-II3.

II. Zhang P, Gu Z, Li WH: Different evolutionary patterns between young duplicate genes in the human genome. Genome Biol 2003, 4:R56.

12. Sonnhammer EL, Koonin EV: Orthology, paralogy and proposed classification for paralog subtypes. Trends Genet 2002 1 8:619-620.

13. Remm M, Storm CE, Sonnhammer EL: Automatic clustering of orthologs and in-paralogs from pairwise species comparisons. J Mol Biol 200I, 3 I4:104I-1052.

14. Kamath RS, Fraser AG, Dong Y, Poulin G, Durbin R, Gotta M, Kanapin A, Le Bot N, Moreno S, Sohrmann M, Welchman DP, Zipperlen $P$, Ahringer J: Systematic functional analysis of the Caenorhabditis elegans genome using RNAi. Nature 2003, 42 I:23 I-237.

15. Piano F, Schetter AJ, Morton DG, Gunsalus KC, Reinke V, Kim SK, Kemphues KJ: Gene clustering based on RNAi phenotypes of ovary-enriched genes in C. elegans. Curr Biol 2002 12:1959-1964

16. Sugimoto A: High-throughput RNAi in Caenorhabditis elegans: genome-wide screens and functional genomics. Differentiation 2004, 72:8I-9I.

17. Hwang HY, Olson SK, Esko JD, Horvitz HR: Caenorhabditis elegans early embryogenesis and vulval morphogenesis require chondroitin biosynthesis. Nature 2003, 423:439-443.

18. Granneman S, Gallagher JE, Vogelzangs J, Horstman W, van Venrooi WJ, Baserga SJ, Pruijn GJ: The human Imp3 and Imp4 proteins form a ternary complex with $\mathrm{hMppl0}$, which only interacts with the U3 snoRNA in $60-80 S$ ribonucleoprotein complexes. Nucleic Acids Res 2003, 3 I: 1877-1887.

19. Bartee E, Mansouri M, Hovey Nerenberg BT, Gouveia K, Fruh K Downregulation of major histocompatibility complex class I by human ubiquitin ligases related to viral immune evasion proteins. J Virol 2004, 78: I 109- I I 20.

20. Deweindt C, Albagli O, Bernardin F, Dhordain P, Quief S, Lantoine D, Kerckaert JP, Leprince D: The LAZ3/BCL6 oncogene encodes a sequence-specific transcriptional inhibitor: a novel function for the BTB/POZ domain as an autonomous repressing domain. Cell Growth Differ 1995, 6: I495-1503.
21. Huynh KD, Bardwell V]: The BCL-6 POZ domain and other POZ domains interact with the co-repressors $\mathbf{N}-\mathbf{C o R}$ and SMRT. Oncogene 1998, I 7:2473-2484.

22. Wong CW, Privalsky ML: Components of the SMRT corepressor complex exhibit distinctive interactions with the POZ domain oncoproteins PLZF, PLZF-RARalpha, and BCL-6. Biol Chem 1998, 273:27695-27702.

23. Xu L, Yang L, Hashimoto K, Anderson M, Kohlhagen G, Pommier $Y$, D'Arpa P: Characterization of BTBDI and BTBD2, two similar BTB-domain-containing Kelch-like proteins that interact with Topoisomerase I. BMC Genomics 2002, 3: I.

24. Xu L, Yang L, Moitra PK, Hashimoto K, Rallabhandi P, Kaul S, Meroni G, Jensen JP, Weissman AM, D'Arpa P: BTBDI and BTBD2 colocalize to cytoplasmic bodies with the RBCC/tripartite motif protein, TRIM5delta. Exp Cell Res 2003, 288:84-93.

25. Yeo M, Lin PS, Dahmus ME, Gill GN: A novel RNA polymerase II C-terminal domain phosphatase that preferentially dephosphorylates serine 5. J Biol Chem 2003, 278:26078-26085.

26. Thompson JD, Higgins DG, Gibson TJ: CLUSTAL W: improving the sensitivity of progressive multiple sequence alignment through sequence weighting, position-specific gap penalties and weight matrix choice. Nucleic Acids Res 1994, 22:4673-4680.

27. Galtier N, Gouy M, Gautier C: SEAVIEW and PHYLO WIN: two graphic tools for sequence alignment and molecular phylogeny. Comput Appl Biosci 1996, 12:543-548.

28. Simmer F, Tijsterman M, Parrish S, Koushika SP, Nonet ML, Fire A Ahringer J, Plasterk RH: Loss of the putative RNA-directed RNA polymerase RRF-3 makes C. elegans hypersensitive to RNAi. Curr Biol 2002, I2:1317-1319.

29. Hobert O: PCR fusion-based approach to create reporter gene constructs for expression analysis in transgenic $C$. elegans. Biotechniques 2002, 32:728-730.

30. Wu W, Hodges E, Redelius J, Hoog C: A novel approach for evaluating the efficiency of siRNAs on protein levels in cultured cells. Nucleic Acids Res 2004, 32:e I7.
Publish with Bio Med Central and every scientist can read your work free of charge

"BioMed Central will be the most significant development for disseminating the results of biomedical research in our lifetime. "

Sir Paul Nurse, Cancer Research UK

Your research papers will be:

- available free of charge to the entire biomedical community

- peer reviewed and published immediately upon acceptance

- cited in PubMed and archived on PubMed Central

- yours - you keep the copyright
BioMedcentral 